\title{
Solidity of viscous liquids
}

\author{
Jeppe C. Dyre \\ Department of Mathematics and Physics (IMFUFA), Roskilde University, P.O. Box 260, DK-4000 Roskilde, Denmark
}

(Received 21 January 1998; revised manuscript received 17 August 1998)

\begin{abstract}
Recent NMR experiments on supercooled toluene and glycerol by Hinze and Böhmer show that small rotation angles dominate with only a few large molecular rotations. These results are here interpreted by assuming that viscous liquids are solidlike on short length scales. A characteristic length, the "solidity length," separates solidlike behavior from liquidlike behavior. [S1063-651X(99)00102-6]
\end{abstract}

PACS number(s): 64.70.Pf, 61.18.Fs, 62.10.+s, 62.90.+k

The viscosity of a liquid approaching the glass transition $[1-12]$ is typically a factor $10^{15}$ larger than the viscosity of ordinary liquids like room-temperature water or ethanol. Although viscosity is just a parameter entering the NavierStokes equation believed to describe any liquid close to equilibrium, this enormous difference raises the question: Are viscous liquids qualitatively different from ordinary liquids or is the difference just quantitative? Below, it is argued that the former is the case. The idea is that viscous liquids behave like solids on short length scales. It is shown that this leads to a prediction consistent with the results of recent NMR experiments by Hinze and Böhmer $[13,14]$.

In many phenomenological models of viscous liquids [3,15-24] flow proceeds via sudden reorientations of molecules, "flow events," which are rare because of large energy barriers to be overcome $[1,17,18,21]$. Kauzmann referred to flow events as "jumps of molecular units of flow between different positions of equilibrium in the liquid's quasicrystalline lattice" [1]. It is this point of view that is explored here: Most molecular motion is purely vibrational and in the time between two flow events a viscous liquid is in a state of elastic equilibrium, just like a solid. However, elastic equilibrium only persists on a certain length scale beyond which the liquid does not display solidlike behavior (this point is returned to below).

Recently, Hinze and Böhmer studied reorientation of toluene and glycerol molecules by means of two-dimensional time-domain NMR spectroscopy $[13,14]$. The rotation angle distribution is dominated by small angles with a small, but significant fraction of larger rotation angles. These findings were interpreted as follows [14]. The large-angle rotations are those required to cross a local energy barrier. Upon barrier crossing local strains are created. These strains are relaxed through small positional and angular adjustments, not only by the molecules in the immediate vicinity but also by those further away. Briefly, large-angle rotations are "causes" and small-angle rotations are "effects." Accepting this picture, we now proceed to show that the rotation angle distribution for small angles may be calculated from the fact that viscous liquids have slow density fluctuations, assuming "solidity" of viscous liquids on short length scales.

First consider density fluctuations. Viscous liquids have long average relaxation times (roughly proportional to viscosity according to Maxwell's relation). The average relaxation time is basically the time between two flow events involving the same molecule. Not only enthalpy or shear stress relaxes on this time scale, but so does density. This has been known for many years from the fact that glass has smaller compressibility than equilibrium viscous liquid. More recently, measurements of the frequency-dependent bulk modulus of viscous liquids [25] revealed a loss peak around the inverse of the Maxwell relaxation time; via the fluctuation-dissipation theorem this shows directly that there are slow density fluctuations. Slow density fluctuations in viscous liquids are probably closely linked to the existence of "dynamic heterogeneities" [26]. Dynamic heterogeneities have been observed, e.g., in light scattering experiments [27,28], NMR experiments [29], time resolved optical spectroscopy [30], and computer simulations [31].

As mentioned, slow density fluctuations take place on a time scale basically determined by the rate of flow events. A flow event is a rapid reorientation of molecules, probably lasting just a few picoseconds. After a flow event the molecules involved have different relative orientations. In most cases, density also changes at the place of a flow event (this is the cause of slow density fluctuations). As a simple model, assume isotropic flow events involving molecules confined to a sphere of radius $r_{0}$ before the flow event. We now proceed to calculate the rotation angle probability distribution for small angles. The induced movement of the surroundings is calculated by means of solid elasticity theory. If the change of radius is $\Delta r$, the displacement of the surroundings is given [32-34] by $u_{x}=\Delta r\left(r / r_{0}\right)^{-2}$, where $r$ is the distance to the flow event. Note that this implies a distribution of displacements varying as $P(u)=P(r)|d r / d u| \propto r^{2} r^{3} \propto u^{-5 / 2}$ $(u \rightarrow 0)$. The average rotation angle $\phi$ is proportional to the strain tensor, which in turn is formed from first order derivatives of $u_{x}$. Consequently, $\phi \propto r^{-3}$ [a detailed calculation gives $\left\langle\phi^{2}\right\rangle=(6 / 5) r_{0}^{4}(\Delta r)^{2} r^{-6}$ ]. The rotation angle probability distribution is given by $P(\phi)=P(r)|d r / d \phi|$. Since $|d r / d \phi| \propto r^{4}$ we find $P(\phi) \propto r^{6}$. Thus $P$ is given by

$$
P(\phi) \propto \phi^{-2} \quad(\phi \rightarrow 0) .
$$

Presently, it is not possible to determine $P(\phi)$ accurately from experiments. It should be noted, though, that since $\sin (\phi) \simeq \phi$ for small $\phi$, Eq. (1) is consistent with the rotation angle distribution tentatively inferred from NMR experiments on glycerol, $P(\phi) \propto 1 / \sin ^{2}(\phi)$ [14].

The rotation angle distribution Eq. (1) is not normalizable, reflecting the fact that in the above derivation all molecules 
of the liquid rotate slightly following a single flow event. This, however, is not realistic; there is a "solidity length", $l$, beyond which flow events effectively do not induce molecular rotations. To estimate $l$ note that elastic displacements propagate with the velocity of sound, $c$. Consider a sphere with radius $R$. Within this sphere there are $N=\left(R / r_{0}\right)^{3}$ possible locations for flow events. A molecule at the center of the sphere only "feels" the full effects from a flow event within the sphere if the following condition is obeyed: The displacement deriving from such a flow event must propagate throughout the sphere and elastic equilibrium be reestablished before another flow event occurs. If $\tau$ is the average relaxation time, the average time between two flow events within the sphere is $\tau / N=\tau\left(R / r_{0}\right)^{-3}$. This time must be longer than or equal to $R / c$. To estimate the solidity length $l$ we use equality for $R=l$ and note that $c$ is, of course, the sound velocity of the glassy state, $c_{\text {glass }}$. This leads to

$$
l^{4}=r_{0}^{3} \tau c_{\text {glass }} .
$$

The solidity length diverges slowly as $\tau \rightarrow \infty$. To get a feeling of the order of magnitude of $l$, consider the case where $\tau=1$ s. Assuming $r_{0}=5 \AA$ and $c_{\text {glass }}=2000 \mathrm{~m} / \mathrm{s}$ one finds $l \simeq 7000 \AA$.

To conclude, below the glass transition, of course, viscous liquids are solid for all practical purposes. It has been argued here that even above the glass transition do viscous liquids in certain respects behave more like solids than like lessviscous liquids. This "solidity" of viscous liquids is limited to length scales below the solidity length $l$. Note that $l$ diverges when $\tau$ diverges; however, $l$ is unrelated to the AdamGibbs characteristic length that also diverges with $\tau$ [17]. Rather, $l$ is similar to the length scale "related to solidlike behavior' recently discussed by Ahluwalia and Das within ideal mode-coupling theory [35], a length scale representative of the distance over which the liquid has enough structure to sustain propagating shear waves. However, the exact relation between the length discussed by Ahluwalia and Das and the solidity length remains to be determined.

This work was supported by the Danish Natural Science Research Council.
[1] W. Kauzmann, Chem. Rev. 43, 219 (1948).

[2] G. Harrison, The Dynamic Properties of Supercooled Liquids (Academic Press, New York, 1976).

[3] S. Brawer, Relaxation in Viscous Liquids and Glasses (American Ceramic Society, Columbus, OH, 1985).

[4] G. P. Johari, in Relaxations in Complex Systems, edited by K. L. Ngai and G. B. Wright (U. S. GPO, Washington, DC, 1985), p. 17.

[5] J. Jäckle, Rep. Prog. Phys. 49, 171 (1986).

[6] C. A. Angell, J. Phys. Chem. Solids 49, 863 (1988).

[7] G. W. Scherer, J. Non-Cryst. Solids 123, 75 (1990).

[8] C. A. Angell, J. Non-Cryst. Solids 131-133, 13 (1991).

[9] A. Hunt, J. Non-Cryst. Solids 160, 183 (1993).

[10] C. A. Angell, Science 267, 1924 (1995).

[11] U. Mohanty, Adv. Chem. Phys. 89, 89 (1995).

[12] M. D. Ediger, C. A. Angell, and S. R. Nagel, J. Phys. Chem. 100, 13200 (1996).

[13] G. Hinze, Phys. Rev. E 57, 2010 (1998).

[14] R. Böhmer and G. Hinze, J. Chem. Phys. 109, 241 (1998).

[15] J. H. Gibbs and E. A. DiMarzio, J. Chem. Phys. 28, 373 (1958).

[16] M. H. Cohen and D. Turnbull, J. Chem. Phys. 31, 1164 (1959).

[17] G. Adam and J. H. Gibbs, J. Chem. Phys. 43, 139 (1965).

[18] M. Goldstein, J. Chem. Phys. 51, 3728 (1969).

[19] G. Williams, in Dielectric and Related Molecular Processes,
Specialist Periodical Report, Vol. 2, edited by M. Davies (Chemical Society, London, 1975), p. 151.

[20] E. Donth, Glasübergang (Akademie-Verlag, Berlin, 1981).

[21] F. H. Stillinger, J. Chem. Phys. 89, 6461 (1988).

[22] R. V. Chamberlin, Phys. Rev. B 48, 15638 (1993).

[23] J. C. Dyre, Phys. Rev. B 51, 12276 (1995).

[24] D. Kivelson, G. Tarjus, X. Zhao, and S. A. Kivelson, Phys. Rev. E 53, 751 (1996).

[25] T. Christensen and N. B. Olsen, Phys. Rev. B 49, 15396 (1994).

[26] D. N. Perera and P. Harrowell, J. Chem. Phys. 104, 2369 (1996).

[27] C. T. Moynihan, and J. Schroeder, J. Non-Cryst. Solids 160, 52 (1993)

[28] C. H. Wang and E. W. Fischer, J. Chem. Phys. 105, 7316 (1996).

[29] R. Böhmer, G. Hinze, G. Diezemann, B. Geil, and H. Sillescu, Europhys. Lett. 36, 55 (1996).

[30] M. T. Cicerone, F. R. Blackburn, and M. D. Ediger, J. Chem. Phys. 102, 471 (1995).

[31] M. M. Hurley and P. Harrowell, Phys. Rev. E 52, 1694 (1995).

[32] L. D. Landau and E. M. Lifshitz, Theory of Elasticity (Pergamon Press, Oxford, 1970).

[33] G. Wyllie, Phys. Rep. 61, 327 (1980).

[34] J. C. Dyre, J. Non-Cryst. Solids 235, 142 (1998).

[35] R. Ahluwalia and S. P. Das, Phys. Rev. E 57, 5771 (1998). 